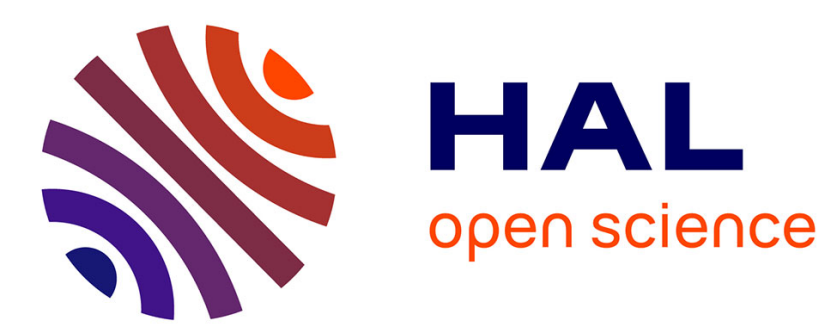

\title{
Prediction-based control with delay estimation of LTI systems with input-output delays
}

Yang Deng, Vincent Léchappé, Emmanuel Moulay, Franck Plestan

\section{To cite this version:}

Yang Deng, Vincent Léchappé, Emmanuel Moulay, Franck Plestan. Prediction-based control with delay estimation of LTI systems with input-output delays. 2019 IEEE ACC, Jul 2019, Philadelphia, United States. pp.3702-3707, 10.23919/ACC.2019.8814876 . hal-02024069

\section{HAL Id: hal-02024069 \\ https://hal.science/hal-02024069}

Submitted on 18 Feb 2019

HAL is a multi-disciplinary open access archive for the deposit and dissemination of scientific research documents, whether they are published or not. The documents may come from teaching and research institutions in France or abroad, or from public or private research centers.
L'archive ouverte pluridisciplinaire HAL, est destinée au dépôt et à la diffusion de documents scientifiques de niveau recherche, publiés ou non, émanant des établissements d'enseignement et de recherche français ou étrangers, des laboratoires publics ou privés. 


\title{
Prediction-based control with delay estimation of LTI systems with input-output delays
}

\author{
Yang Deng, Vincent Léchappé, Emmanuel Moulay and Franck Plestan
}

\begin{abstract}
The aim of this article is to propose a predictionbased controller combined with a new time-delay estimation method for LTI systems with unknown input and output delays. The global asymptotic stability of the time-delay system is ensured. The proposed control scheme includes a delay estimator which estimates the unknown round-trip delay (the sum of input and output delays), a Luenberger observer and a prediction-based controller. Among the main results of this article, the delay estimator is firstly introduced; secondly, a Lyapunov-Razumikhin analysis is given to prove the stability of the closed-loop system; finally, several examples are given to illustrate the performances of the proposed method.
\end{abstract}

Keywords: Time-delay systems; Delay estimation; LyapunovRazumikhin theorem

\section{INTRODUCTION}

Time-delay system (TDS) is a wide research domain in the control community since time-delays are very common in real systems. For example, time-delays can arise from communication protocol, remote control and sensor measurement [1]. Input and output delays are important to consider because they can degrade performances or even destabilize systems [2]. This article deals with the system with unknown constant input and output delays which appear for instance in networked control systems [3]. In real systems, it is very difficult to directly measure the unknown input and output delays or separately estimate them, then a round-trip delay estimation is proposed in this article.

A vast literature is available on the control of input-delay systems. Finite spectrum assignment (FSA) [4] and reduction method [5] are standard methods to deal with known inputdelays. Prediction-based control is also an efficient way to stabilize the TDS with known input-delays, it is used in [6] for linear systems and in [7] for nonlinear systems. For the systems with unknown input-delays, the works in [8], [9], [10] present a backstepping partial differential equation (PDE) transformation method. The new system is a combination of an ordinary differential equation (ODE) and a PDE transport equation. Therefore, the adaptive controller design and Lyapunov-Krasovskii analysis are proposed to stabilize the ODE-PDE system. Besides, a large number of works without PDE transformation have been done to deal with the unknown input-delay. Several approaches among them

Yang Deng and Franck Plestan are with Ecole Centrale de Nantes, LS2N UMR CNRS 6004, Nantes, France. Emmanuel Moulay is with Université de Poitiers, XLIM UMR CNRS 7252, Poitiers, France. Vincent Léchappé is with INSA de Lyon, AMPERE UMR CNRS 5005, Lyon, France. (e-mail: yang.deng@1s2n.fr, vincent.lechappe@insa-lyon.fr, emmanuel.moulay@univ-poitiers.fr, franck.plestan@ec-nantes.fr). Corresponding author: Yang Deng. are based on the time-delay estimation (TDE) algorithm. A modified Smith predictor with transfer function based TDE algorithm is provided in [11]. The authors of [12] propose a memory-free controller combined with a piecewise-constant input delay identifier for LTI systems with an input-delay. The exponential stability of the closed-loop system is obtained but the delay identifiability of the closed-loop system must be ensured. The works in [13] provide an open-loop TDE algorithm and a robust predictive controller with an application to a DC motor, the TDS is stabilized in practice.

However, the systems with input and output delays are not as widely studied as input-delay systems. The works in [14] present an advanced reduction method which stabilizes the linear systems with known constant input, output and state delays. A predictor-based controller for nonlinear systems with zero-order-hold input and sampled measurement is proposed in [15], this work is extended to output feedback controller in [16]. In [17], a truncated predictor feedback approach is proposed to stabilize the systems with known input and output time-varying delays, but this technique only deals with the systems with particular poles. An adaptive controller for hyper-minimum-phase systems with uncertain input and output delays is proposed in [18], but the drawback is that the controller only ensures the local stability and it cannot deal with arbitrarily long delays.

In this article, a real-time round-trip delay estimation method is combined with a Luenberger observer and a prediction-based controller. It is shown that if the initial condition of the delay estimator is close enough to the round-trip delay value, then the asymptotic stability of the TDS can be obtained. The stability condition is obtained by using Lyapunov-Razumikhin ${ }^{1}$ method. Thus, one obtains the stabilization of the system and the convergence of the state estimation.

The article is organized as follows. The problem statement is presented in Section II. Then, the main results are provided in Section III and illustrated by simulation results in Section IV. Finally, a conclusion is given in Section V.

\section{Problem StATEMEnT}

In this article, the following notations are used. Let $\|x\|$ denote the euclidean norm of a vector $x \in \mathbb{R}^{n}$. The matrix $I_{n}$ represents the identity matrix of size $n \times n$. The matrix norm of a matrix $A$ reads as $\|A\|$. The maximum and minimum eigenvalues of the matrix $A$ are respectively defined as $\bar{\lambda}(A)$

\footnotetext{
${ }^{1}$ The details of the Lyapunov-Razumikhin theorem are presented in $[2$, Subsection 3.1.3] and [19, Subsection 5.4].
} 
and $\underline{\lambda}(A)$. Let $\mathbf{C}=C\left([\sigma-h, \sigma+r], \mathbb{R}^{n}\right)$ for any $t \in[\sigma, \sigma+$ $r]$, then $x_{t}(\theta)$ can be defined as $x_{t}(\theta)=x(t+\theta) \in \mathbf{C}$ with $-h \leq \theta \leq 0$. The other notations of the Lyapunov stability are given in [19].

Consider a LTI system with input and output delays

$$
\begin{cases}\dot{x}(t) & =A x(t)+B u\left(t-h_{i}\right) \\ y(t) & =C x\left(t-h_{o}\right)\end{cases}
$$

where $x(t) \in \mathbb{R}^{n}, A \in \mathbb{R}^{n \times n}, B \in \mathbb{R}^{n \times p}, C \in \mathbb{R}^{m \times n}, u(t) \in \mathbb{R}^{p}$ and $h_{i}, h_{o}$ are unknown constant time-delays. Therefore, the round-trip delay reads as

$$
h_{i o}=h_{i}+h_{o}
$$

which is bounded in $\left[h_{\min }, h_{\max }\right]$. The boundedness $h_{\max }>$ $h_{\min }>0$ are assumed to be known. The initial condition of system (1) is defined as $x(\theta)=x_{0}(\theta) \in \mathbf{C}$ and $u(\theta)=$ $u_{0}(\theta) \in \mathbf{C}$ for all $\theta \in\left[-2 h_{\max }, 0\right]$.

A Luenberger observer presented in $[6, \mathrm{p} .61]$ is proposed to estimate the output-delayed state $x\left(t-h_{o}\right)$ :

$$
\dot{\hat{x}}(t)=A \hat{x}(t)+B u\left(t-h_{i o}\right)+L[y(t)-C \hat{x}(t)]
$$

with initial condition $\hat{x}(\theta)=\hat{x}_{0}(\theta) \in \mathbf{C}$ for all $\theta \in$ $\left[-2 h_{\text {max }}, 0\right]$. The estimation error is defined as

$$
e(t)=\hat{x}(t)-x\left(t-h_{o}\right)
$$

An approximated prediction ${ }^{2} z(t)$ based on $\hat{h}(t)$ and $\hat{x}(t)$ used in [10] is proposed in this article

$$
z(t)=e^{A \hat{h}} \hat{x}(t)+\int_{t-\hat{h}}^{t} e^{A(t-s)} B u(s) \mathrm{d} s
$$

with initial condition $z(\theta)=z_{0}(\theta) \in \mathbf{C}$ for all $\theta \in$ $\left[-2 h_{\max }, 0\right]$.

The problem to solve is to design the dynamics of $\dot{\hat{h}}(t)$ and a controller $u(z(t))$, in order to obtain the asymptotic stability of the closed-loop system.

In the sequel the following assumptions are made.

Assumption 1. The pair $(A, B)$ is stabilizable and the pair $(A, C)$ is observable.

Assumption 1 ensures that the controller and observer can be designed. It follows that there exists feedback matrices $K, L$ with appropriate dimensions such that $A+B K, A-L C$ are Hurwitz, and for all positive constants $c_{u}, c_{l}$, there exists symmetric positive definite matrices $P \in \mathbb{R}^{n \times n}, Q \in \mathbb{R}^{n \times n}$ that are the solutions of the following Lyapunov equations

$$
\begin{aligned}
& (A+B K)^{T} P+P(A+B K)=-c_{u} I_{n} \\
& (A-L C)^{T} Q+Q(A-L C)=-c_{l} I_{n} .
\end{aligned}
$$

Assumption 2. The delayed input value $u\left(t-h_{i o}\right)$ is known.

In practical application, it is possible to save the delayed input value $u\left(t-h_{i}\right)$ and send it to the controller along with

\footnotetext{
${ }^{2}$ The predictor-based controller contains infinite dimensional term which may cause implementation issues. To well implement this controller, a trapezoid discretization method and a periodic reset of the update law when convergence is achieved are proposed in [10].
}

the system's output. Therefore, as a result of the transport delay $h_{o}$, one obtains $u\left(t-h_{i}-h_{o}\right)$. The structure of the closed-loop system is shown in Figure 1. The information sent from the controller to the system and from the system to the controller are subject to the unknown input and output delays. The blue dashed rectangle represents the control scheme that includes the observer, the predictorbased controller and the delay estimator. The violet dashed rectangle represents the communication delays introduced by the communication channels.

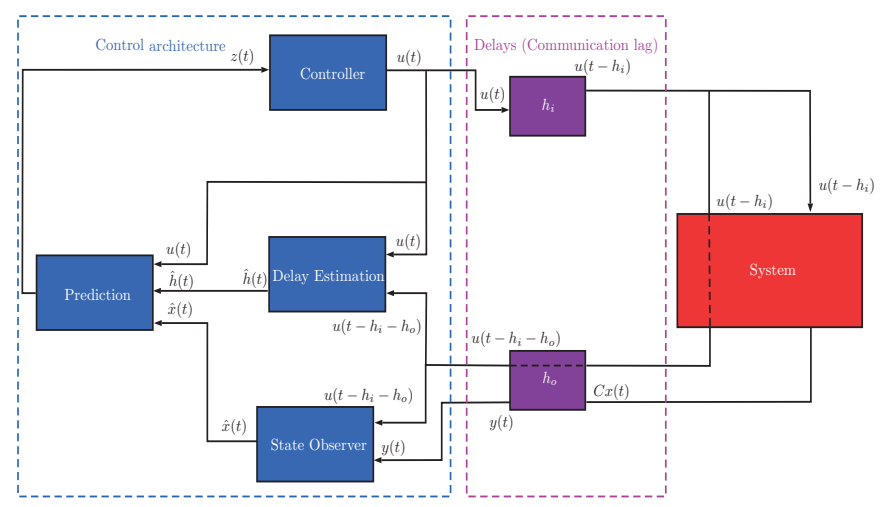

Fig. 1. Closed-loop scheme of the prediction-based controller with TDE algorithm.

\section{DELAY ESTIMATION AND STABILIZATION OF THE INPUT-OUTPUT DELAY SYSTEM}

The main result of this paper is given by Theorem 1 .

Theorem 1. Consider that the system (1) satisfies Assumptions 1 and 2, the observer (3) and the predictor (5). The control law satisfies

$$
\begin{aligned}
u(t) & =K z(t), \quad t>0 \\
u(\theta) & =u_{0}(\theta)=K z_{0}(\theta), \quad \theta \in\left[-2 h_{\text {max }}, 0\right]
\end{aligned}
$$

where $K$ is the linear feedback matrix referred in Assumption 1. Suppose that the control input $u(t)$ given in (8) is continuously differentiable at $t=0$. Define the delay estimator

$$
\left\{\begin{array}{l}
\dot{\hat{h}}(t)=\min \left\{\gamma\left\|u\left(t-h_{i o}\right)-u(t-\hat{h}(t))\right\|, \hat{\delta}\right\}, \quad t>0 \\
\hat{h}(\theta)=\hat{h}_{0}, \quad \theta \in\left[-2 h_{\max }, 0\right]
\end{array}\right.
$$

where $\gamma, \hat{\delta}$ are positive constants and the initial condition $\hat{h}_{0}$ satisfies $h_{\text {min }} \leq \hat{h}_{0} \leq h_{i o}$. If $\hat{h}_{0}$ is sufficiently close to $h_{i o}$, then there exists a constant $\hat{\delta}^{*}>0$, such that for all $\hat{\delta}<\hat{\delta}^{*}$, the z-system is uniformly globally asymptotically stable, then one gets

$$
\begin{aligned}
& \lim _{t \rightarrow+\infty}\|x(t)\|=0 ; \\
& \lim _{t \rightarrow+\infty}\|e(t)\|=0 .
\end{aligned}
$$

Proof: The proof is divided into 3 parts. The first part studies the properties of delay estimator (9); the second part proves the stability of the closed-loop $z$-system; the last part demonstrates the convergence of $x(t)$ to zero by using the stability of the $z$-system and the convergence of $e(t)$. 
Step 1. In this step, the properties of the delay estimator $\hat{h}(t)$ are studied. The delay estimation satisfies the following inequalities

$$
\hat{h}_{0} \leq \hat{h}(t) \leq h_{i o}
$$

for all $t \geq 0$. Inequalities (12) are hereafter proven by contradiction. Assume that there exists $t_{0}>0$ such that $\hat{h}\left(t_{0}\right)>h_{i o}$. From equation (9), it follows that $\dot{\hat{h}}(t) \geq 0$ for all $t \geq-2 h_{\max }$. Therefore, $\hat{h}(t)$ is continuous, monotonically increasing with initial condition $\hat{h}_{0} \leq h_{i o}$ for all $\theta \in\left[-2 h_{\text {max }}, 0\right]$. The intermediate value theorem given in [20, p.105] implies that there exists $t_{1} \in\left(0, t_{0}\right)$ such that $\hat{h}\left(t_{1}\right)=h_{i o}$ and $\hat{\hat{h}}\left(t_{1}\right)>0$. However, if $\hat{h}\left(t_{1}\right)=h_{i o}$, then one has

$$
\dot{\hat{h}}\left(t_{1}\right) \leq \gamma\left\|u\left(t-h_{i o}\right)-u\left(t-\hat{h}\left(t_{1}\right)\right)\right\|=0 .
$$

Therefore, inequality (13) contradicts the fact $\dot{\hat{h}}\left(t_{1}\right)>0$ and the existence of $t_{0}$. Then, inequalities (12) are proven by contradiction.

This step ensures that if the initial condition of the delay estimator satisfies

$$
h_{i o}-\hat{h}_{0} \leq D
$$

with $D>0$, then the delay estimator $\hat{h}(t)$ satisfies

$$
0 \leq h_{i o}-\hat{h}(t) \leq h_{i o}-\hat{h}_{0} \leq D
$$

for all $t \geq 0$ due to the monotonicity and the boundedness of $\hat{h}(t)$.

Step 2. Differentiating equation (5) for all $t>0$ and substituting $\dot{\hat{x}}(t)$ by equation (3), one obtains

$$
\begin{aligned}
\dot{z}(t)= & (A+B K) z(t)+\dot{\hat{h}}(t) A z(t)+\dot{\hat{h}}(t) e^{A \hat{h}(t)} B u(t-\hat{h}(t)) \\
& -\dot{\hat{h}}(t) A \int_{t-\hat{h}(t)}^{t} e^{A(t-s)} B u(s) d s-e^{A \hat{h}(t)} L C e(t) \\
& +e^{A \hat{h}(t)} B\left[u\left(t-h_{i o}\right)-u(t-\hat{h}(t))\right] .
\end{aligned}
$$

Developing system (1) at $t-h_{o}$ yields that

$$
\dot{x}\left(t-h_{o}\right)=A x\left(t-h_{o}\right)+B u\left(t-h_{o}-h_{i}\right) .
$$

Noticing that $h_{o}$ is constant, it implies that

$$
\frac{d}{d t} x\left(t-h_{o}\right)=\dot{x}\left(t-h_{o}\right)
$$

Differentiating the estimation error $e(t)$ by using equations (3), (17) and (18), it leads to

$$
\begin{aligned}
\dot{e}(t)= & \dot{\hat{x}}(t)-\frac{d}{d t} x\left(t-h_{o}\right) \\
= & A \hat{x}(t)+B u\left(t-h_{i o}\right)-L C e(t) \\
& \quad-A x\left(t-h_{o}\right)-B u\left(t-h_{o}-h_{i}\right) \\
= & (A-L C) e(t) .
\end{aligned}
$$

Define $V_{1}(z(t))=z(t)^{T} P z(t), V_{2}(e(t))=e(t)^{T} Q e(t)$. From equation (9), it yields that $|\hat{h}(t)|$ is upper bounded by $\hat{\delta}$ for all $t \geq-2 h_{\max }$. Differentiating $V_{1}(z(t))$ along the trajectories of (16), it leads to

$$
\begin{aligned}
\dot{V}_{1}(z(t)) \leq & -c_{u}\|z(t)\|^{2}+2 \hat{\delta}\|P A\|\|z(t)\|^{2} \\
& +2 \hat{\delta} e^{\|A\| h_{\max }}\|B\|\|P\|\|z(t)\|\|u(t-\hat{h}(t))\| \\
& +2 \hat{\delta} e^{\|A\| h_{\max }}\|P A\|\|B\|\|z(t)\|\|v(t)\| \\
& +2 e^{\|A\| h_{\max }}\|P\|\|L C\|\|z(t)\|\|e(t)\| \\
& +2 e^{\|A\| h_{\max }}\|P\|\|B\|\|z(t)\|\|w(t)\|
\end{aligned}
$$

where $\quad\|v(t)\|=\int_{t-\hat{h}(t)}^{t}\|u(s)\| \mathrm{d} s \quad$ and $\quad\|w(t)\|=$ $\int_{t-h_{i o}}^{t-\hat{h}(t)}\|\dot{u}(s)\| \mathrm{d} s . \quad$ Differentiating $\quad V_{2}(e(t)) \quad$ along the trajectories of (19), one obtains

$$
\dot{V}_{2}(e(t))=-c_{l}\|e(t)\|^{2} .
$$

The following Lyapunov-Razumikhin candidate function is defined by

$$
V(z(t), e(t))=V_{1}(z(t))+\alpha V_{2}(e(t))
$$

where $\alpha$ is a positive constant. Defining $c_{1}=2\|P A\|$, $c_{2}=2 e^{\|A\| h_{\max }}\|B\|\|P\|, \quad c_{3}=2 e^{\|A\| h_{\max }}\|P A\|\|B\|$ and $c_{4}=$ $2 e^{\|A\| h_{\max }}\|P\|\|L C\|$, the time-derivative of $V(z(t), e(t))$ satisfies

$$
\begin{aligned}
\dot{V}(z(t), e(t)) \leq & -\left(c_{u}-\hat{\delta} c_{1}\right)\|z(t)\|^{2}+\hat{\delta} c_{2}\|z(t)\|\|u(t-\hat{h}(t))\| \\
& +\hat{\delta} c_{3}\|z(t)\|\|v(t)\|+c_{4}\|z(t)\|\|e(t)\| \\
& +c_{2}\|z(t)\|\|w(t)\|-\alpha c_{l}\|e(t)\|^{2} .
\end{aligned}
$$

Therefore, one can use Lyapunov-Razumikhin condition as follows: for a given $\kappa>1$, suppose that one has

$$
V(z(t-s), e(t-s)) \leq \kappa V(z(t), e(t)), \quad s \in\left[0,2 h_{\max }\right]
$$

and therefore one has

$$
\begin{aligned}
& \|z(t-s)\| \leq c_{5}(\|z(t)\|+\sqrt{\alpha}\|e(t)\|), \quad s \in\left[0,2 h_{\text {max }}\right] \\
& \|e(t-s)\| \leq c_{5}^{*}(\|z(t)\| / \sqrt{\alpha}+\|e(t)\|), \quad s \in\left[0,2 h_{\text {max }}\right]
\end{aligned}
$$

where $\quad c_{5}=\sqrt{\kappa \max (\bar{\lambda}(P), \bar{\lambda}(Q)) / \underline{\lambda}(P)} \quad$ and $\quad c_{5}^{*}=$ $\sqrt{\kappa \max (\bar{\lambda}(P), \bar{\lambda}(Q)) / \underline{\lambda}(Q)}$. Thus, one can simplify the second and third terms of inequality (23) as follows:

$$
\begin{aligned}
\hat{\delta} c_{2}\|z(t)\|\|u(t-\hat{h}(t))\| \leq & \hat{\delta} c_{2} c_{5}\|K\|\|z(t)\|(\|z(t)\|+\sqrt{\alpha}\|e(t)\|) \\
& \leq \hat{\delta} c_{6}\|z(t)\|(\|z(t)\|+\sqrt{\alpha}\|e(t)\|)
\end{aligned}
$$




$$
\begin{array}{r}
\hat{\delta} c_{3}\|z(t)\|\|v(t)\| \leq \hat{\delta} c_{3} c_{5}\|K\| h_{\max }\|z(t)\|(\|z(t)\|+\sqrt{\alpha}\|e(t)\|) \\
\leq \hat{\delta} c_{7}\|z(t)\|(\|z(t)\|+\sqrt{\alpha}\|e(t)\|)
\end{array}
$$

where $c_{6}=c_{2} c_{5}\|K\|$ and $c_{7}=c_{3} c_{5}\|K\| h_{\max }$. To simplify the term $c_{2}\|z(t)\|\|w(t)\|$ of inequality (23), one uses

$$
\|w(t)\| \leq D(t)\|K\| \sup _{s \in\left[t-h_{i o}, t-\hat{h}(t)\right]}\|\dot{z}(s)\|
$$

with $D(t)=h_{i o}-\hat{h}(t)$. For all $s_{1} \in\left[\hat{h}(t), h_{i o}\right]$, one can develop the expression of $\dot{z}\left(t-s_{1}\right)$ by using equation (16):

$$
\begin{aligned}
\dot{z}\left(t-s_{1}\right)= & (A+B K) z\left(t-s_{1}\right)+\dot{\hat{h}}\left(t-s_{1}\right) A z\left(t-s_{1}\right) \\
& -\dot{\hat{h}}\left(t-s_{1}\right) A \int_{t-s_{1}-\hat{h}\left(t-s_{1}\right)}^{t-s_{1}} e^{A\left(t-s_{1}-s\right)} B u(s) \mathrm{d} s \\
& +\dot{\hat{h}}\left(t-s_{1}\right) e^{A \hat{h}\left(t-s_{1}\right)} B u\left(t-s_{1}-\hat{h}\left(t-s_{1}\right)\right) \\
& +e^{A \hat{h}\left(t-s_{1}\right)} B\left[u\left(t-s_{1}-h_{i o}\right)-u\left(t-s_{1}-\hat{h}\left(t-s_{1}\right)\right)\right] \\
& -e^{A \hat{h}\left(t-s_{1}\right)} L C e\left(t-s_{1}\right) .
\end{aligned}
$$

Taking norm of equation (29), by using the Razumikhin condition (25) and the triangle inequality, it leads to

$$
\begin{aligned}
\left\|\dot{z}\left(t-s_{1}\right)\right\| & \leq\|A+B K\| c_{5}(\|z(t)\|+\sqrt{\alpha}\|e(t)\|) \\
& +\hat{\delta}\|A\| c_{5}(\|z(t)\|+\sqrt{\alpha}\|e(t)\|) \\
& +\hat{\delta}\|A\| e^{\|A\| h_{\max }}\|B K\| h_{\max } c_{5}(\|z(t)\|+\sqrt{\alpha}\|e(t)\|) \\
& +\hat{\delta} e^{\|A\| h_{\max }}\|B K\| c_{5}(\|z(t)\|+\sqrt{\alpha}\|e(t)\|) \\
& +2 e^{\|A\| h_{\max }}\|B K\| c_{5}(\|z(t)\|+\sqrt{\alpha}\|e(t)\|) \\
& +e^{\|A\| h_{\max }}\|L C\| c_{5}^{*}(\|z(t)\| / \sqrt{\alpha}+\|e(t)\|)
\end{aligned}
$$

for all $s_{1} \in\left[\hat{h}(t), h_{i o}\right]$. Inequality (30) is equivalent to

$$
\begin{aligned}
\sup _{s \in\left[t-h_{i o}, t-\hat{h}(t)\right]}\|\dot{z}(s)\| \leq & \left(c_{8}(\hat{\boldsymbol{\delta}})+c_{9} / \sqrt{\alpha}\right)\|z(t)\| \\
& +\left(c_{8}(\hat{\boldsymbol{\delta}}) \sqrt{\alpha}+c_{9}\right)\|e(t)\|
\end{aligned}
$$

with parameters $c_{8}(\hat{\boldsymbol{\delta}})$ and $c_{9}$ defined by

$$
\begin{aligned}
c_{8}(\hat{\boldsymbol{\delta}})= & \|A+B K\| c_{5}+\hat{\boldsymbol{\delta}}\|A\| c_{5} \\
& +e^{\|A\| h_{\max }}\|B K\|\left(2+\hat{\boldsymbol{\delta}}+\hat{\boldsymbol{\delta}}\|A\| h_{\max }\right) c_{5}, \\
c_{9}= & e^{\|A\| h_{\max }}\|L C\| c_{5}^{*} .
\end{aligned}
$$

Substituting inequality (31) into inequality (28), and using inequality (15) one maximizes $c_{2}\|z(t)\|\|w(t)\|$ as:

$$
\begin{aligned}
c_{2}\|z(t)\|\|w(t)\| \leq & D(t)\left(c_{10}(\hat{\delta})+c_{11} / \sqrt{\alpha}\right)\|z(t)\|^{2} \\
& +D(t)\left(c_{10}(\hat{\delta}) \sqrt{\alpha}+c_{11}\right)\|z(t)\|\|e(t)\| \\
& \leq D\left(c_{10}(\hat{\delta})+c_{11} / \sqrt{\alpha}\right)\|z(t)\|^{2} \\
& +D\left(c_{10}(\hat{\delta}) \sqrt{\alpha}+c_{11}\right)\|z(t)\|\|e(t)\|
\end{aligned}
$$

with $c_{10}(\hat{\boldsymbol{\delta}})=c_{2} c_{8}(\hat{\boldsymbol{\delta}})\|K\|, c_{11}=c_{2} c_{9}\|K\|$ and $D=h_{i o}-\hat{h}_{0}$. Therefore, inequality (23) can be simplified as

$$
\begin{aligned}
\dot{V}(z(t), e(t)) & \leq-\left(c_{u}-\hat{\delta} c_{12}-D c_{10}(\hat{\delta})-\frac{D c_{11}}{\sqrt{\alpha}}\right)\|z(t)\|^{2} \\
& +\left(\hat{\delta} c_{13} \sqrt{\alpha}+c_{4}+D c_{10}(\hat{\delta}) \sqrt{\alpha}\right. \\
& \left.+D c_{11}\right)\|z(t)\|\|e(t)\|-\alpha c_{l}\|e(t)\|^{2}
\end{aligned}
$$

with $c_{12}=c_{1}+c_{6}+c_{7}$ and $c_{13}=c_{6}+c_{7}$. Finally, one obtains the following inequality by completing the squares:

$$
\begin{aligned}
\dot{V}(z(t), e(t)) \leq & -\left(c_{u}-\hat{\delta} c_{12}-D c_{10}(\hat{\boldsymbol{\delta}})-\frac{D c_{11}}{\sqrt{\alpha}}-\frac{\hat{\delta} c_{13} \sqrt{\alpha}}{2}\right. \\
& \left.-\frac{D c_{10}(\hat{\boldsymbol{\delta}}) \sqrt{\alpha}}{2}-\frac{D c_{11}}{2}-\frac{c_{4}^{2}}{2 \alpha c_{l}}\right)\|z(t)\|^{2} \\
& -\alpha\left(\frac{c_{l}}{2}-\frac{D c_{10}(\hat{\boldsymbol{\delta}})}{2 \sqrt{\alpha}}-\frac{D c_{11}}{2 \alpha}-\frac{\hat{\delta} c_{13}}{2 \sqrt{\alpha}}\right)\|e(t)\|^{2} .
\end{aligned}
$$

Choosing $\alpha$ sufficiently large so that $\frac{c_{4}^{2}}{2 \alpha c_{l}}$ can be ignored by comparing with $c_{u}$, then there exists sufficiently small constants $\hat{\delta}^{*}$ and $D^{*}$ such that

$$
\begin{gathered}
c_{u}-\hat{\delta}^{*} c_{12}-D^{*} c_{10}\left(\hat{\delta}^{*}\right)-\frac{D^{*} c_{11}}{\sqrt{\alpha}}-\frac{\hat{\delta}^{*} c_{13} \sqrt{\alpha}}{2} \\
-\frac{D^{*} c_{10}\left(\hat{\delta}^{*}\right) \sqrt{\alpha}}{2}-\frac{D^{*} c_{11}}{2} \geq 0, \\
\frac{c_{l}}{2}-\frac{D^{*} c_{10}\left(\hat{\delta}^{*}\right)}{2 \sqrt{\alpha}}-\frac{D^{*} c_{11}}{2 \alpha}-\frac{\hat{\delta}^{*} c_{13}}{2 \sqrt{\alpha}} \geq 0 .
\end{gathered}
$$

If the initial condition of the delay estimator is sufficiently close to $h_{i o}$ such that $h_{i o}-\hat{h}_{0}=D<D^{*}$ is ensured. Then, for all $\hat{\delta}<\hat{\delta}^{*}$, there exists two constants $c_{14}, c_{15}>0$ such that

$$
\dot{V}(z(t), e(t)) \leq-c_{14}\|z(t)\|^{2}-c_{15}\|e(t)\|^{2}
$$

which implies that there exists a nondecreasing continuous function $w(s): \mathbb{R}^{+} \rightarrow \mathbb{R}^{+}$such that

$$
\dot{V}(z(t), e(t)) \leq-w(z(t), e(t)) .
$$

The Lyapunov-Razumikhin theorem referred in [19, Subsection 5.4, Theorem 4.2] ensures that the closed-loop $z$-system is uniformly globally asymptotically stable. Therefore, the convergence results $\lim _{t \rightarrow \infty}\|z(t)\|=0$ and $\lim _{t \rightarrow \infty}\|e(t)\|=0$ are also ensured.

Step 3. As proven in Step 2, one obtains $\lim _{t \rightarrow \infty}\|z(t)\|=0$ and $\lim _{t \rightarrow \infty}\|e(t)\|=0$. Reminding that equation (5) equals to

$$
\hat{x}(t)=e^{-A \hat{h}(t)} z(t)-\int_{t-\hat{h}(t)}^{t} e^{A(t-\hat{h}(t)-s)} B u(s) \mathrm{d} s .
$$


Reminding the definition of $e(t)$, equation (39) can be developed as

$$
x\left(t-h_{o}\right)=e^{-A \hat{h}(t)} z(t)-e(t)-\int_{t-\hat{h}(t)}^{t} e^{A(t-\hat{h}(t)-s)} B u(s) \mathrm{d} s .
$$

As known that $0<\hat{h}(t) \leq h_{\max }$, by using the mean value theorem referred in [20, p. 101], one bounds the integral term of equation (40) as follows:

$$
\begin{aligned}
\left\|\int_{t-\hat{h}(t)}^{t} e^{A(t-\hat{h}(t)-s)} B u(s) \mathrm{d} s\right\| & \leq e^{\|A\| h_{\max }}\|B\| \int_{t-\hat{h}(t)}^{t}\|u(s)\| \mathrm{d} s \\
& \leq e^{\|A\| h_{\max }}\|B\| \int_{t-h_{i o}}^{t}\|u(s)\| \mathrm{d} s \\
& \leq e^{\|A\| h_{\max }}\|B\|\|K\| h_{\max } \sup _{s \in\left[t-h_{i o}, t\right]}\|z(s)\|
\end{aligned}
$$

for all $t \geq h_{i o}$. Taking the norm of equation (40) and using the triangle inequality, it leads to

$$
\begin{aligned}
\left\|x\left(t-h_{o}\right)\right\| & \leq e^{\|A\| h_{\max }}\|z(t)\|+\|e(t)\| \\
& +e^{\|A\| h_{\max }}\|B\|\|K\| h_{\max } \sup _{s \in\left[t-h_{i o}, t\right]}\|z(s)\| .
\end{aligned}
$$

Noticing that the limit of the right-hand side of inequality (42) is zero as $t$ tends towards infinity because of the convergences $\lim _{t \rightarrow \infty}\|z(t)\|=0$ and $\lim _{t \rightarrow \infty}\|e(t)\|=0$, then one obtains $\lim _{t \rightarrow \infty}\|x(t)\|=0$.

Remark 1. Delay estimator (9) is the key point of the stabilization of system (1). It is not necessary for the delay estimator to converge to the nominal round-trip delay since the system can be stabilized with delay mismatch [12], [21]. However, in applications, the unknown round-trip delay can be estimated by delay estimator (9) as it will be shown in simulations.

Remark 2. In theory, it is necessary to set $\hat{\delta}$ sufficiently small to ensure that the stability condition (36) always holds. Indeed, Theorem 1 presents only a sufficient condition for the stability of the closed-loop system. Although condition (36) is not satisfied with larger $\hat{\delta}$, the convergence $\lim _{t \rightarrow \infty}\|z(t)\|=0$ ultimately holds with higher convergence speed of the TDE algorithm in applications.

Remark 3. Delay estimator (9) is always increasing, if the initial condition $\hat{h}_{0}$ is not carefully chosen such that $\hat{h}_{0} \geq h_{i o}$, then the delay estimation and the system will diverge. In practice, it is possible to set $\hat{h}_{0}=h_{\text {min }}$ in order to avoid the divergence.

Remark 4. Theorem 1 also shows that the predictor-based controller is robust with respect to the slight delay mismatch.
If one uses a constant estimation $\bar{h}$ that is sufficiently close to $h$ instead of $\hat{h}(t)$ to calculate the control law given in (5) and (8). Then the same proof as Theorem 1 can be given to guarantee that the closed-loop system is asymptotically stable. This result is very similar to the one given in [21].

Remark 5. The proposed TDE method is robust with respect to the model uncertainties and the external disturbances. The reason is that the control input $u(t)$ is memorized and the delayed input value $u\left(t-h_{i o}\right)$ is measured, then the delay estimator will not be influenced by the model uncertainties and the external disturbances.

\section{Simulations}

Two simulations of a second-order system are made to evaluate the results provided in Theorem 1. Moreover, the delay estimator converges to the round-trip delay in simulations.

\section{A. Simulation results with $\hat{h}_{0}$ close to $h_{\text {io }}$}

Consider the second-order system

$$
\begin{cases}\dot{x}(t) & =A x(t)+B u\left(t-h_{i}\right) \\ y(t) & =C x\left(t-h_{o}\right)\end{cases}
$$

with $A=\left[\begin{array}{cc}0.5 & 1 \\ 2.5 & -1\end{array}\right], \quad B=\left[\begin{array}{l}0 \\ 1\end{array}\right], \quad C=\left[\begin{array}{ll}1 & 0\end{array}\right], \quad x(\theta)=$ $\left[\begin{array}{ll}0.4 & 1\end{array}\right]^{T}$ for all $\theta \in\left[-2 h_{\max }, 0\right]$ and $z(\theta)=\hat{x}(\theta)=\left[\begin{array}{ll}0 & 0\end{array}\right]^{T}$ for all $\theta \in\left[-2 h_{\max }, 0\right]$. The unknown time-delays are set to $h_{i}=0.5 \mathrm{~s}, h_{o}=0.3 \mathrm{~s}$, the initial condition of the delay estimator is set to $\hat{h}_{0}=0.7 \mathrm{~s}$, the gain $\gamma=5$ and the derivative of delay estimator (9) is bounded by $\hat{\delta}=0.1$. The parameters of the controller are defined as $K=\left[\begin{array}{ll}-7.7 & -3.35\end{array}\right]$ and $L=\left[\begin{array}{ll}4.5 & 2.5\end{array}\right]^{T}$.

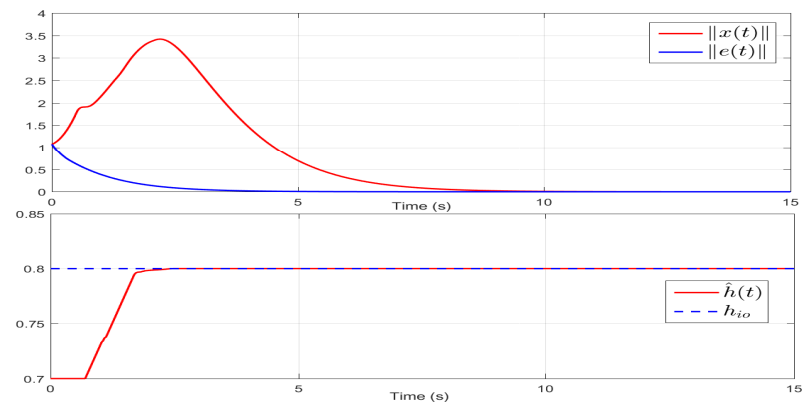

Fig. 2. Evolution of $\|x(t)\|,\|e(t)\|$ and $\hat{h}(t)$ versus time for system (43) with $\hat{h}_{0}=0.7 \mathrm{~s}$. 
The simulation results are presented in Figure 2. The unknown round-trip delay is well estimated and one gets $\lim _{t \rightarrow \infty}\|e(t)\|=0, \lim _{t \rightarrow \infty}\|x(t)\|=0$.

Figure 2 also verifies the discussions in Remark 2, i.e. the fact that the theoretical stability condition (36) can only be satisfied with sufficiently small $\hat{\delta}$ because of the conservatism. Indeed, the convergence of system (43) holds when $\delta^{*}=0.1$ whereas the theoretical bound is around $10^{-4}$ in order that condition (36) can be ensured.

\section{B. Simulation results with $\hat{h}_{0}$ far from $h_{\text {io }}$}

Another simulation is done for the same system (43), except $\hat{h}_{0}$ that has been redefined as $\hat{h}_{0}=0.2 \mathrm{~s}$. The simulation

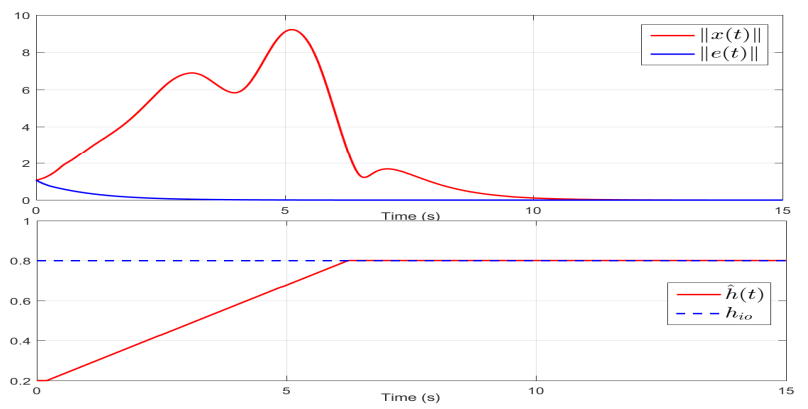

Fig. 3. Evolution of $\|x(t)\|,\|e(t)\|$ and $\hat{h}(t)$ versus time for system (43) with $\hat{h}_{0}=0.2 \mathrm{~s}$.

results are presented in Figure 3. The unknown round-trip delay is also well estimated, and one has the convergences $\lim _{t \rightarrow \infty}\|e(t)\|=0, \lim _{t \rightarrow \infty}\|x(t)\|=0$. Since the initial condition of $\hat{h}(t)$ is far from $h_{i o}$, there exists a transient for the delay estimator to be sufficiently close to the round-trip delay. As the delay estimation $\hat{h}(t)$ gets sufficiently close to $h_{i o}$ after $t=6 \mathrm{~s}$, the convergences of $\|x(t)\|$ and $\|e(t)\|$ to zero ultimately hold. This simulation shows that the constraints on the initial condition of $\hat{h}(t)$ can be relaxed in practice.

\section{CONCLUSION}

In this article, a new round-trip delay estimation method for constant input and output delays is proposed. The stability of the closed-loop system is guaranteed by LyapunovRazumikhin theorem. The practical implementation of this approach is discussed, and the theoretical results are illustrated by simulations. The extension of this method to nonlinear systems and to time-varying delays will be considered for future works.

\section{ACKNOWLEDGEMENTS}

This work is a part of the Ph.D. thesis of Yang Deng, who is supported by China Scholarship Council (CSC).

\section{REFERENCES}

[1] J. Chiasson and J. J. Loiseau, Applications of time delay systems, ser. Lecture Notes in Control and Information Sciences. Springer, 2007, vol. 352.

[2] E. Fridman, Introduction to time-delay systems: Analysis and control, ser. Systems \& Control: Foundations \& Applications. Birkhäuser, 2014.

[3] M. Garca-Rivera and A. Barreiro, "Analysis of networked control systems with drops and variable delays," Automatica, vol. 43, no. 12, pp. $2054-2059,2007$

[4] A. Manitius and A. Olbrot, "Finite spectrum assignment problem for systems with delays," IEEE Transactions on Automatic Control, vol. 24, no. 4, pp. 541-552, 1979.

[5] Z. Artstein, "Linear systems with delayed controls: A reduction," IEEE Transactions on Automatic control, vol. 27, no. 4, pp. 869-879, 1982.

[6] I. Karafyllis and M. Krstic, Predictor feedback for delay systems: Implementations and approximations. Springer, 2017.

[7] F. Cacace, F. Conte, A. Germani, and P. Pepe, "Stabilization of strict-feedback nonlinear systems with input delay using closed-loop predictors," International Journal of Robust and Nonlinear Control, vol. 26, no. 16, pp. 3524-3540, 2016.

[8] M. Krstic, Delay compensation for nonlinear, adaptive, and PDE systems, ser. Systems \& Control: Foundations \& Applications. Birkhäuser, 2009.

[9] N. Bekiaris-Liberis and M. Krstic, "Delay-adaptive feedback for linear feedforward systems," Systems \& Control Letters, vol. 59, no. 5, pp. 277-283, 2010

[10] D. Bresch-Pietri, J. Chauvin, and N. Petit, "Adaptive control scheme for uncertain time-delay systems," Automatica, vol. 48, no. 8, pp. $1536-1552,2012$

[11] J. Herrera and A. Ibeas, "On-line delay estimation for stable, unstable and integrating systems under step response," ISA Transactions, vol. 51, no. 3, pp. 351-361, 2012.

[12] F. Cacace, F. Conte, and A. Germani, "State feedback stabilization of linear systems with unknown input time delay," IFAC-PapersOnLine, vol. 50, no. 1, pp. 1245 - 1250, 2017, 20th IFAC World Congress.

[13] V. Léchappé, S. Rouquet, A. Gonzalez, F. Plestan, J. De León, E. Moulay, and A. Glumineau, "Delay estimation and predictive control of uncertain systems with input delay: Application to a DC motor," IEEE Transactions on Industrial Electronics, vol. 63, no. 9, pp. 5849-5857, 2016.

[14] M. Jankovic, "Recursive predictor design for state and output feedback controllers for linear time delay systems," Automatica, vol. 46, no. 3 , pp. $510-517,2010$.

[15] I. Karafyllis and M. Krstic, "Stabilization of nonlinear delay systems using approximate predictors and high-gain observers," Automatica, vol. 49, no. 12, pp. 3623 - 3631, 2013

[16] I. Karafyllis, M. Krstic, T. Ahmed-Ali, and F. Lamnabhi-Lagarrigue, "Global stabilisation of nonlinear delay systems with a compact absorbing set," International Journal of Control, vol. 87, no. 5, pp. 1010-1027, 2014

[17] B. Zhou, Z.-Y. Li, and Z. Lin, "Observer based output feedback control of linear systems with input and output delays," Automatica, vol. 49, no. 7, pp. 2039-2052, 2013

[18] A. Selivanov, E. Fridman, and A. Fradkov, "Passification-based adaptive control: Uncertain input and output delays," Automatica, vol. 54, pp. 107-113, 2015.

[19] J. K. Hale and S. M. V. Lunel, Introduction to functional differential equations, ser. Applied Mathematical Sciences. Springer, 1993, vol. 99.

[20] R. Beals, Analysis: an introduction. Cambridge University Press, 2004.

[21] D. Bresch-Pietri and N. Petit, "Prediction-based control for linear systems with input-and state-delay-robustness to delay mismatch," in IFAC World Congress 2014, 2014, pp. 11410-11418. 